BULL. AUSTRAL. MATH. SOC.

VOL. $23(1981), 321-338$.

\title{
NONLINEAR HYDROMAGNETIC CONVECTION \\ IN A MODERATE PRANDTL NUMBER FLUID
}

\author{
N. RIAHI
}

\begin{abstract}
Nonlinear hydromagnetic connection is investigated using the modal equations for cellular convection. The boundary layer method is used assuming large Rayleigh number $R$, moderate Prandtl number $\sigma$ and for different ranges of the Chandrasekhar number $Q$. The heat flux $F$ is determined for the value of the horizontal wave number which maximizes $F$. For a weak field, the inertial force dominates over the Lorentz force. $F$ is independent of $Q$, but it increases with $R$ and $\sigma$. For a moderate field, the Lorentz force is significant. $F$ increases with $R$ and $\sigma$ and decreases as $Q$ increases. For a strong field, the Lorentz force dominates over the inertial force. $F$ is independent of $\sigma$, but it increases with $R$ and decreases as $Q$ increases.
\end{abstract}

\section{Introduction}

The specific problem considered in this paper is that of the effect of an imposed uniform magnetic field on a nonlinear convection in a moderate Prandtl number $\sigma$ fluid at large Rayleigh numbers with $\tau=\frac{\text { magnetic diffusivity }}{\text { thermal diffusivity }} 1$. Hydromagnetic convection is important in many areas of geophysics and astrophysics. An example in geophysics is the convective motion in the earth's fluid core ( $\tau \gg 1, \sigma \leq 1)$ which is believed to be responsible for driving the geodynamo. The purpose of the present investigation is to provide us with further insights into the

Received 25 November 1980. 
subject of nonlinear hydromagnetic convection in situations where $\tau$ is large and $\sigma$ is moderate, rather than provide a quantitative comparison with particular natural situations.

We shall study nonlinear magnetic convection at moderate Prandtl numbers under the so called modal equations of the equations for momentum, heat and magnetic field. Briefly, these equations are constructed by expanding the fluctuating quantities in a complete set of functions of the horizontal coordinate, and then truncating the expansion. The single mode equations are derived simply by retaining only the first term in the expansion. For a more detailed discussion of these equations and their derivations, we refer to the paper by Gough et al. [4]. The same system of equations has been derived earlier, in a different manner, by Roberts [7] using a procedure proposed by Glansdorff and Prigogine [3]. Numerical computations of the single mode equations for thermal convection have recently been carried out by Toomre et al. [9], and good agreement with the observation and with the esymptotic results obtained by Gough et al. [4] was found.

Recently, Riahi [6] studied nonlinear magnetic convection under so called mean-field equations of the equations for momentum, heat and magnetic field. These equations are derived by ignoring the interaction between the fluctuation quantities but retaining the interaction between the mean and the fluctuation quantities. The single mode mean-field equations are identical to the modal equations (2.1a - 2.1c) given in the next section when the parameter $c$ is set to zero. Since the equation of motion in the mean-field approximation $(c=0)$ becomes identical to the equation of motion in the limit of infinite Prandtl number $\sigma$, it must be expected that the mean-field theory is most realistic in the case of large $\sigma$. The modal equations for $c \neq 0$ has some representation of the missing nonlinear interactions of fluctuating quantities in the mean-field equations and has the advantage that it restores the Prandtl number as a parameter of the problem. In their studies of cellular convection, Gough et al. [4] compared their solutions based on the modal equations with the known solutions of the full equations for moderate Rayleigh numbers (Malkus and Veronis, [5]; Schluter, Lortz, and Busse, [8]). They found that accuracy is restricted to plan-forms for which $c \neq 0$ and otherwise to $\sigma \gg 1$. Therefore, the modal equations $(c \neq 0)$ are particularly 
suitable for the study of cellular convection in a moderate Prandtl number fluid.

In the present study, we are interested in finding the solution which maximizes the heat transport $F$. The flow that maximizes $F$ determines uniquely the horizontal wave number and gives an upper bound on the actual $F$. Although, in general, the $F$ that maximizes for the modal hydromagnetic equations is not necessarily that $F$ which maximizes for the original full hydromagnetic convection equations (for $\tau \gg l$ ), it is expected in light of previous upper bound studies, that the upper bound on $F$ based on the modal equations represents adequately the upper bound on $F$ based on the full Boussinesq hydromagnetic convection equations, provided the Rayleigh number, though large, is restricted and bounded from above. The success of the previous upper bound studies of thermal convection which have determined interesting features and similarities with observation as well as useful bounds on $F$, encouraged us to undertake the present study. The reader is also referred to Gough et al. [4] for a detailed discussion in support of the studies based on the single-mode equations for cellular convection.

The treatment here is for the steady case. Numerical studies by Weiss [10] indicate that a steady state can be reached by a hydromagnetic convective flow of finite amplitude. Of course, sufficiently strong convective flows are time dependent, but the present study aims at exploring the properties of nonlinear magnetic convection in the simpler case of a steady state, which may be considered as an approximation in some sense.

Before we start formulating the problem and presenting the technical details, it is useful to place a brief physical discussion of the results for the flow conditions with various external field strength. When the imposed vertical field is weak the convection and the heat transport $F$ are unaffected and the fluctuating field is produced kinematically. The Lorentz force is small and can be neglected from the momentum equation. For a moderate field the Lorentz force is significant and partially reduces the effects of inertial force on $F$ so that $F$ depends weakly on $\sigma$. The convective cells reduce in horizontal size and $F$ is reduced. For a strong field the Lorentz force is so large that it dominates over the inertial force so that $F$ becomes independent of $\sigma$. The rigidity which 
is imparted by the external field tends to suppress the convection and the heat transport is seriously reduced.

\section{Mathematical Formulation}

We consider an infinite horizontal layer of fluid of depth $d$ permeated by a uniform magnetic field of strength $M$ in the vertical direction. The upper and lower surfaces are assumed to be stress free and are maintained at temperatures $T_{0}$ and $T_{0}+\Delta T(\Delta T>0)$, respectively. The modal equations of the hydromagnetic convection are derived from the Boussinesq equations for momentum, magnetic field and heat by expanding the fluctuating variables in the planform functions $F_{n}(x, y)$ of linear theory. The reader is referred to the paper by Gough et al. [4] for a detailed derivation of the modal equations for thermal convection. The nondimensional steady state forms of these equations, after eliminating pressure and truncating the expansion by retaining only the first terms are:

(2.1a) $\left(D^{2}-a^{2}\right)^{2} W=R a^{2} T+c \sigma^{-1}\left[2 D W\left(D^{2}-a^{2}\right) W+W\left(D^{2}-a^{2}\right) D W\right]-\tau Q\left(D^{2}-a^{2}\right) D h_{3}$,

$$
\tau\left(D^{2}-a^{2}\right) h_{3}+D W=0
$$

$$
\left(D^{2}-a^{2}\right) T+(1-W T+F) W=C(2 W D T+T D W) .
$$

The advection of the fluctuating magnetic field $h$ in (2.1b) and the nonlinear interactions of $h$ with $h$ and velocity vector $u$ in (2.la, b) are neglected, since the present analysis is restricted to the parameter range

$$
\tau \gg 1,
$$

where $\tau=\frac{\eta}{K}$ is the ratio of magnetic diffusivity to thermal diffusivity. In the above equations, $h_{3} F_{1}$ and $\omega F_{1}$ are the vertical components of $h$ and $u$ respectively, $T F_{1}$ is the deviation of the temperature from its horizontal average, $R=\alpha g \Delta T d^{3} / k \nu$ is the Rayleigh number, $Q=\frac{M^{2} d^{2}}{\mu \rho \nu n}$ is the Chandrasenkhar number, $\sigma=\frac{\nu}{K}$ is the Prandtl number, $\mu$ is the 
magnetic permeability, $\rho$ is the reference density (constant). $v$ is the kinematic viscosity, $\alpha$ is the coefficient of thermal expansion, and $g$ the acceleration due to gravity. Also, $a$ is the horizontal wave number, $D=d / d z, F=\langle W T\rangle$ is the heat flux, $c=\frac{1}{\frac{1}{2} F_{1}^{3}(x, y)}$ is the parameter derived from the planform function $F_{1}(x, y)$, the bars denote horizontal average, and the angle brackets denote a further vertical average over the whole layer. The constant $c$ vanishes for rolls and rectangles and takes the value of $6^{-\frac{1}{2}}$ for the hexagonal planform. We shall assume $c \neq 0$ and consider the value such as $6^{-\frac{3}{2}}$ as representative value of $c$. For $c=0$, the system (2.1) reduces to the so called mean field equations for the magnetic convection, and the problem has recently been solved (Riah $i$ [6]).

It is convenient to rescale the dependent variables so that

$$
\omega=(F R)^{-\frac{3}{2}} W, \quad \theta=(R / F)^{\frac{3}{2}} T, \quad H=(F R)^{-\frac{1}{2}} \tau h_{3} .
$$

The governing equations then take the following forms:

(2.4a) $\left(D^{2}-a^{2}\right)^{2} \omega=a^{2} \theta+c(F R)^{\frac{1}{2}} \sigma^{-1}\left[2 D \omega\left(D^{2}-a^{2}\right) \omega+\omega\left(D^{2}-a^{2}\right) D \omega\right]-Q\left(D^{2}-a^{2}\right) D H$,

$$
\left(D^{2}-a^{2}\right) H+D w=0 \text {, }
$$

$$
(F R)^{-1}\left(D^{2}-a^{2}\right) \theta+\left(1-\omega \theta+F^{-1}\right) \omega=c(F R)^{-\frac{1}{2}}(2 \omega D \dot{\theta}+\theta D \omega) .
$$

We shall use the following constraint to evaluate the heat transport

$$
F=\frac{1-R^{-1}\left(|\nabla \theta|^{2}\right)}{\left((1-\omega \theta)^{2}\right)},
$$

which is obtained by multiplying $(2.4 \mathrm{c})$ by $\theta$ and taking the vertical average over the layer.

The boundary conditions to be considered for the free surfaces at $z=0,1$ are

$$
\omega=D^{2} \omega=\theta=H=0 .
$$

The subsequent analysis and solution of (2.4)-(2.6) supposes throughout that both $R$ and $F$ are large. The magnitude of $Q$ varies, and different classes of solutions are found for different orders of magnitude 
of it. In each case the principle focus is on the unique solution that maximizes $F$.

\section{Solutions by boundary layer method}

\subsection{THE CASE OF A WEAK FIELD}

The wave number $a$ is supposed to be large (which can be justified $a$ posteriori), so that the convection cells are narrow. The solutions can be obtained by matching asymptotic approximations in interior and three distinct regions near each boundary. Without loss of generality, we shall restrict ourselves to the discussion of the boundary layer structure near the lower boundary, since the boundary layer structures near the upper and lower boundaries are essentially identical.

In the interior of the layer, $z$ is of order one. It is assumed that

$$
a^{4} \ll F R \ll a^{6} \sigma^{2},
$$

$$
Q \ll a^{4} .
$$

The governing equations $(2.4 a)-(2.4 c)$ yield, after using (3.1), the following equations

$$
\begin{aligned}
a^{2} \omega & =\theta, \\
\omega \theta & =1, \\
a^{2} H & =D \omega .
\end{aligned}
$$

$(3.2 a)-(3.2 c)$ yield the following solutions

$$
\begin{aligned}
& \omega=a^{-1}, \\
& \theta=a, \\
& H=0 .
\end{aligned}
$$

Near the boundary and adjacent to the interior is an inertial layer, in which inertial terms are significant. We define $\phi=z / \varepsilon$ as the variable in the layer, where $\varepsilon$ denotes the thickness of the layer. We then find from (2.4), after applying matching conditions (matching the solutions to the corresponding solutions in the interior), that the equations in the inertial layer are 
$(3.4 a)$

$$
a^{2} \omega=\theta-3 a^{3} \omega \frac{d \omega}{d \phi},
$$

$$
\omega \theta=1 \text {, }
$$

$$
\varepsilon \alpha^{2} H=\frac{d \omega}{d \phi},
$$

where it is found appropriate to assume that

$$
\varepsilon=c \sigma^{-1} a^{-3}(F R)^{\frac{1}{2}} \gg a^{-1},
$$

$$
Q \ll a^{4} \varepsilon^{2} .
$$

The solution to $(3.4 a)$ satisfying the boundary condition $w=0$ at $\phi=0$ satisfies the following equation

$$
-3 a \omega+\log \left(\frac{1+\alpha \omega}{1-\alpha \omega}\right)^{3 / 2}=\phi \text {. }
$$

$(3.4 \mathrm{~b}, \mathrm{c})$ and $(3.6)$ yield the following asymptotic results

$$
\begin{aligned}
\omega & =a^{-1} \phi^{1 / 3}, \\
\theta & =a \phi^{-1 / 3}, \\
3 H & =\epsilon^{-1} a^{-3} \phi^{-2 / 3}
\end{aligned}
$$

as $\phi \rightarrow 0$. Closer to the boundary and adjacent to the inertial layer is an intermediate layer of thickness $a^{-1}$, in which vertical derivatives are important in the momentum advection terms. Defining $\xi=a z$ as the variable in this layer, (2.4) and matching conditions (matching the solutions to the correcponding solutions in the inertial layer) yield

$$
\theta+\sigma^{-1} a c(F R)^{\frac{1}{2}}\left[2 \frac{d \omega}{d \xi}\left(\frac{d^{2}}{d \xi^{2}}-1\right) \omega+\omega\left(\frac{d^{2}}{d \xi^{2}}-1\right) \frac{d \omega}{d \xi}\right]=0 \text {, }
$$

$$
\omega \theta=1 \text {, }
$$

$$
a\left(\frac{d^{2}}{d \xi^{2}}-1\right) H+\frac{d \omega}{d \xi}=0
$$

where it is assumed that

$$
Q \ll\left(a^{4} \varepsilon\right)^{2 / 3} .
$$

$(3.8 a)-(3.8 c)$ yield the following results 
$(3.10 a)$

$$
\omega=\left(\frac{\sigma}{c a}\right)^{1 / 3}(F R)^{-1 / 6} \xi\left(3 \log \xi^{-1}\right)^{1 / 3}
$$

$(3.10 b)$

$$
\theta=\omega^{-1} \text {, }
$$

as $\xi \rightarrow 0$,

$(3.10 c)$

$$
H=-\left(\frac{1}{2 a}\right)\left(\frac{\sigma}{c a}\right)^{1 / 3}(F R)^{-1 / 6} \xi^{2}\left(3 \log \xi^{-1}\right)^{1 / 3}
$$

as $\xi \rightarrow 0$.

There is a further thinner layer closer to the boundary, in which thermal conduction is significant in the heat equation and $\theta$ is brought to its zero boundary value. We define $\delta$ as the thickness of the layer and $\eta=z / \delta$ as the variable in the layer. The governing equations and matching conditions then give the following equations in the thermal layer:

$$
\frac{d^{4} \omega}{d \eta^{4}}=c \sigma^{-1} \delta(F R)^{\frac{1}{2}}\left(2 \frac{d \omega}{d \eta} \cdot \frac{d^{2} \omega}{d \eta^{2}}+\omega \frac{d^{3} \omega}{d \eta^{3}}\right)-Q \delta \frac{d^{3} H}{d n^{3}},
$$

$$
(F R)^{-1} \delta^{-2} \frac{d^{2} \theta}{d \eta^{2}}+(1-\omega \theta) \omega=c \delta^{-1}(F R)^{-\frac{1}{2}}\left(2 \omega \frac{d \theta}{d \eta}+\theta \frac{d \omega}{d \eta}\right) .
$$

In deriving (3.11), it is found that we must have the following conditions

$(3.12 c)$

$$
\begin{aligned}
F R A^{2} \delta^{2} & =1, \\
a \delta & <1, \\
a^{2} \delta^{4} & \ll \sigma^{-1} A^{2},
\end{aligned}
$$

where

$$
A=\left(\frac{\sigma}{c a}\right)^{1 / 3}(F R)^{-1 / 6} a \delta\left(3 \log \frac{1}{a \delta}\right)^{1 / 3} .
$$

The solutions to $(3.11)$ satisfying $(2.6)$ are

$$
\omega=A \eta,
$$

$$
H=-\left(\frac{1}{2}\right) \delta A n^{2} \text {, }
$$


$(3.13 c)$

$$
\theta=\frac{c^{\frac{1}{2}} \eta}{2 A} \int_{1}^{\mu^{2}}\left(\mu^{2}-t^{2}\right)^{-\frac{1}{2}} \exp \left[\frac{c}{2} \eta^{2}(1-t)\right] d t
$$

where.

$$
\mu^{2}=1+c^{-2} \text {. }
$$

To determine $F$, we must evaluate the expressions $\left\langle|\nabla \theta|^{2}\right)$ and $\left((1-\omega \theta)^{2}\right)$ in (2.5). Within the boundary layer approximation, using the results above and keeping only the leading order terms, we find that

$$
\left(|\nabla \theta|^{2}\right)=a^{4}+2 \delta^{-1} A^{-2} I_{1},
$$

$$
\left((1-\omega \theta)^{2}\right\rangle=2 \delta I_{2}
$$

where $I_{1}$ and $I_{2}$ are the integrals $\int_{0}^{\infty}\left(\frac{d \theta}{d \eta}\right)^{2} d n$ and $\int_{0}^{\infty}(1-n \theta)^{2} d n$ in the thermal layer, respectively. Using (3.14) in (2.5) and maximizing $F$ with respect to $a$, yield the following results

(3.15a) $\quad \varepsilon=\left(\frac{39}{5}\right)^{1 / 10}\left(\frac{6}{I}\right)^{3 / 5}\left(\frac{c}{\sigma}\right)^{9 / 10} R^{-1 / 10}\left[\log \left(\sigma R^{\frac{3}{4}}\right)\right]^{1 / 10}$,

(3.15b) $\quad a=\left(\frac{R}{13}\right)^{\frac{3}{4}}$,

(3.15c) $\delta=\left(\frac{5 I}{18}\right)^{1 / 5}(13)^{3 / 10}\left(\frac{c}{\sigma}\right)^{1 / 5} R^{-3 / 10}\left[\log \left(\sigma R^{\frac{1}{4}}\right)\right]^{-1 / 5}$,

(3.15a) $\quad E=\left(\frac{6}{13 I}\right)^{6 / 5}\left(\frac{3}{5}\right)^{1 / 5}(13)^{-1 / 10}\left(\frac{\sigma}{c}\right)^{1 / 5} R^{3 / 10}\left[\log \left(\sigma R^{\frac{1}{4}}\right)\right]^{1 / 5}$,

where

$(3.15 e)$

$$
I=I_{1}+I_{2}=1.062\left(I+c^{2}\right)^{\frac{1}{4}}
$$

Various assumptions including (3.1), (3.5), (3.9) and (3.12) lead us to the following conditions for the validity of the solutions

$$
\left(R^{-1} \log R\right)^{1 / 6} \ll \sigma \ll R^{1 / 6}(\log R)^{-1},
$$

$$
Q \ll\left(R \sigma^{-1}\right)^{3 / 5}\left[\log \left(\sigma R^{\frac{3}{4}}\right)\right]^{1 / 5} .
$$




\subsection{THE CASE OF A MODERATE FIELD}

The solutions for this case can be obtained by matching asymptotic approximations in the interior and four distinct regions near each boundary. The interior and the inertial layer discussed in the last section are unchanged and (3.1)-(3.7) hold again. Closer to the boundary and adjacent to the inertial layer is a magnetic layer, in which Lorentz force becomes significant and balance the inertial and buoyancy forces. We define $\psi=z / \gamma$ as the variable in this layer, where $\gamma$ denotes the thickness of the layer. The governing equations (2.4) and matching conditions then give the following equations in the magnetic layer:

$$
\begin{gathered}
\gamma \theta-3 c \sigma^{-1}(F R)^{\frac{1}{2}} \omega \frac{d \omega}{d \psi}+Q \frac{d H}{d \psi}=0, \\
\omega \theta=1, \\
\gamma \alpha^{2} H=\frac{d \omega}{d \psi},
\end{gathered}
$$

where it is assumed that

$$
a^{-1} \ll \gamma=\left(\frac{Q}{3 a^{2}}\right)^{3 / 4}\left(\frac{\sigma}{c}\right)^{\frac{3}{2}}(F R)^{-\frac{1}{4}} \ll \varepsilon,
$$

$$
a^{3} \ll \text { FRoY } .
$$

$(3.17 a)-(3.17 c)$ yield the following asymptotic results

(3.19a) $\quad \omega=\left(\frac{\sigma \gamma}{c}\right)^{1 / 3}(F R)^{-1 / 6}\left(\frac{2}{3}\right)^{\frac{3}{2}} \psi\left(\log \psi^{-1}\right)^{\frac{1}{2}}$,

(3.19b) $\quad \theta=w^{-1}$, as $\psi \rightarrow 0$.

(3.19c) $\left.\quad H=\left(\frac{\sigma}{c}\right)^{1 / 3}(F R)^{-1 / 6}\left(\frac{2}{3}\right)^{\frac{1}{2}} a^{-2} \gamma^{-2 / 3}\left(\log \psi^{-1}\right)^{\frac{1}{2}}\right)$

Closer to the boundary and adjacent to the magnetic layer is an intermediate layer of thickness $a^{-1}$. Defining $\xi=a z$ as the variable in this layer, (2.4) and matching conditions yield

$$
\left(\frac{d^{2}}{d \xi^{2}}-1\right) \frac{d H}{d \xi}=0
$$

$$
\omega \theta=1,
$$


$(3.20 c)$

$$
a\left(\frac{d^{2}}{d \xi^{2}}-1\right) H+\frac{d \omega}{d \xi}=0,
$$

where it is assumed that

$$
a^{6} \gamma^{2} \ll F R \sigma(\log a \gamma)^{3 / 2}
$$

$$
a^{2} \ll Q \text {. }
$$

The solutions to $(3.20)$ are

$$
\omega=\left(\frac{\sigma \gamma}{c}\right)^{1 / 3}(F R)^{-1 / 6}\left(\frac{2}{3}\right)^{\frac{1}{2}}(\alpha \gamma)^{-1}(\log \alpha \gamma)^{\frac{1}{2}} \xi
$$

$$
\theta=\omega^{-1} \text {, }
$$

(3.22c) $\quad H=\left(\frac{2}{3}\right)^{\frac{3}{2}}\left(\frac{\sigma}{c}\right)^{1 / 3}(F R)^{-1 / 6} a^{-2} \gamma^{-2 / 3}(\log a \gamma)^{\frac{3}{2}}[1-\exp (-\xi)]$.

There is a further layer closer to the boundary and adjacent to the intermediate layer, in which thermal conduction is significant and $\theta$ is brought to its zero boundary value. Using the same notations as those used in Section 3.1, the governing equations (2.4) and matching conditions yield $(3.11)-(3.12 b),(3.13 a),(3.13 c)-(3.13 d)$ and the following expressions for $A$ and $H$,

$$
A=\left(\frac{\sigma \gamma}{c}\right)^{1 / 3}(F R)^{-1 / 6}\left(\frac{2}{3}\right)^{\frac{1}{2}}\left(\frac{\delta}{\gamma}\right)(\log \alpha \gamma)^{\frac{1}{2}}
$$

$$
H=a^{-1} A \eta \text {. }
$$

The maximization of $F$ proceeds as before, and we find the following asymptotic results

$$
\varepsilon=\left(\frac{4}{I}\right)^{2 / 3}\left(\frac{c}{\sigma}\right) Q^{-1 / 6}\left[\log \left(\sigma R^{-1} Q^{5 / 3}\right)\right]^{1 / 6}
$$

$$
a=\left(\frac{R}{9}\right)^{\frac{3}{4}} \text {, }
$$

$$
\gamma=\left(\frac{T}{4}\right)^{1 / 3}\left(\frac{\sigma}{c}\right)^{\frac{1}{2}}\left(\frac{3}{R}\right)^{3 / 4} Q^{5 / 6}\left[\log \left(\sigma R^{-1} Q^{5 / 3}\right)\right]^{-1 / 12} \text {, }
$$

$(3.24 d)$

$$
\delta=3\left(\frac{I Q}{4}\right)^{1 / 3} R^{-\frac{1}{2}}\left[\log \left(\sigma R^{-1} Q^{5 / 3}\right)\right]^{-1 / 3}
$$


$(3.24 \mathrm{e})$

$$
F=\left(\frac{1}{27}\right)\left(\frac{4}{I}\right)^{4 / 3} R^{\frac{1}{2}} Q^{-1 / 3}\left[\log \left(\sigma R^{-1} Q^{5 / 3}\right)\right]^{1 / 3} .
$$

Various assumptions considered in this section lead to the following conditions for the validity of the solutions

(3.25a) $\left(R \sigma^{-1}\right)^{3 / 5}\left[\log \left(\sigma R^{-1} Q^{5 / 3}\right)\right]^{1 / 10} \ll Q \ll R^{3 / 4}\left[\log \left(\sigma R^{-1} Q^{5 / 3}\right)\right]$

$$
\text { for } R^{-1 / 6}\left[\log \left(\sigma R^{-1} Q^{5 / 3}\right)\right]^{1 / 6} \ll \sigma \leq\left[\log \left(\sigma R^{-1} Q^{5 / 3}\right)\right]^{-\frac{3}{2}} \text {, }
$$

(3.25b) $\left(R \sigma^{-1}\right)^{3 / 5}\left[\log \left(\sigma R^{-1} Q^{5 / 3}\right)\right]^{1 / 10} \ll Q \ll R^{3 / 4} \sigma^{-3 / 2}\left[\log \left(\sigma R^{-1} Q^{5 / 3}\right)\right]^{\frac{1}{4}}$

$$
\text { for }\left[\log \left(\sigma R^{-1} Q^{5 / 3}\right)\right]^{-\frac{1}{2}} \ll \sigma \ll\left[R \log \left(\sigma R^{-1} Q^{5 / 3}\right)\right]^{1 / 6} \text {. }
$$

\subsection{THE CASE OF A MODERATELY STRONG FIELD}

The above boundary layer solutions in the range (3.25) was based essentially on the condition that $a \ll \delta^{-1}$. Now as $Q$ further increases beyond the ranges (3.25), it can be a new condition that $a=\delta^{-1}$. Thus, the thermal layer merges with the intermediate layer. The solutions in this new thermal layer are then found to be

(3.26a) $\omega=A n$,

(3.26b) $\quad H=a^{-1} A[1-\exp (-\eta)]$,

(3.26c) $\theta=\frac{e^{\frac{1}{2}} \eta}{2 A}\left(\frac{\mu+1}{\mu-1}\right)^{1 / 4 c \mu} \int_{1}^{\mu}\left(\frac{\mu-t}{\mu+t}\right)^{1 / 4 c \mu}\left(\mu^{2}-t^{2}\right)^{-\frac{1}{4}} \exp \left[\frac{c}{2} n^{2}(1-t)\right] d t$, where $A$ is defined in (3.23a).

The maximization of $F$ can proceed as before, and we find the following results:

$$
\gamma=2^{7 / 4 \cdot 2} \dot{\sigma}\left(\frac{\sigma}{c}\right)^{\frac{1}{2}}\left(\frac{Q}{3}\right)^{5 / 2} R^{-2}\left[\log \left(Q^{2} R^{-4 / 3} \sigma^{2 / 3}\right)\right]^{-7 / 4}
$$

$$
\begin{aligned}
& \varepsilon=\left(\frac{c}{\sigma}\right)\left(\dot{j} R^{-1}\right)^{2}\left(\frac{2 Q}{3}\right)^{5 / 2}\left[\log \left(Q^{2} R^{-4 / 3} \sigma^{2 / 3}\right)\right]^{-5 / 2}, \\
& a=\left(\frac{3 R}{2 \dot{j} Q}\right)\left[\log \left(Q^{2} R^{-4 / 3} \sigma^{2 / 3}\right)\right],
\end{aligned}
$$




$$
F=\dot{J}^{-2}\left(\frac{3 R}{2 Q}\right)\left[\log \left(Q^{2} R^{-4 / 3} \sigma^{2 / 3}\right)\right]
$$

where

$$
\begin{aligned}
& \text { (3.28) } \dot{J} \simeq-(3 c)^{-1}(2 \pi)^{3 / 2}\left\{\left[\left(\frac{\pi}{2}\right)^{2}(3)^{\frac{1}{2}}(\mu-1)+\frac{3}{2}(\mu+1)-\frac{1}{c}\right]\right. \\
& \cdot\left[1-2^{-1}(3)^{\frac{3}{2}}\right]^{\frac{1}{4}((1 / c \mu)-3)} \cdot\left[\frac{1}{2}(3)^{\frac{1}{2}}(\mu-1)+(3 \mu+1)\right]^{-\frac{1}{4}((1 / c \mu)+5)} \\
& +\left[\frac{2}{2}(\mu+1)-\frac{1}{c}\right](3 \mu+1)^{-\frac{1}{4}((1 / c \mu)+5)}+\left[-\left(\frac{1}{2}\right)^{2}(3)^{\frac{1}{2}}(\mu-1)+\frac{1}{2}(\mu+1)-\frac{1}{c}\right] \\
& \left.\cdot\left[(3)^{\frac{1}{2}} 2^{-1}+1\right]^{\frac{1}{4}((1 / c \mu)-3)} \cdot\left[-2^{-1}(3)^{\frac{1}{2}}(\mu-1)+(3 \mu+1)\right]^{-\frac{1}{4}((1 / c \mu)+5)}\right\} \\
& \cdot(\mu-1)^{-7 / 4}(\mu+1)^{1 / 4 c \mu}
\end{aligned}
$$

Various assumptions considered in this section lead to the following conditions for the validity of the solutions

(3.29a) $R^{3 / 4}\left[\log \left(Q^{2} R^{-4 / 3} \sigma^{2 / 3}\right)\right] \ll Q \ll\left(R^{2} \sigma\right)^{2 / 5}\left[\log \left(Q^{2} R^{-4 / 3} \sigma^{2 / 3}\right)\right]$,

$$
R^{-1 / 8} \ll \sigma \ll\left[\log \left(Q^{2} R^{-4 / 3} \sigma^{2 / 3}\right)\right]^{-\frac{2}{2}} .
$$

\subsection{THE CASE OF A STRONG FIELD}

For $Q$ larger and beyond the range $(3.29 a)$, the condition that the magnetic layer thickness $\gamma$ should satisfy (3.18) no longer holds, and we can then have a new condition in which

$$
a^{-1} \ll \delta \ll 1
$$

The detailed analysis for (3.30) (though omitted here) indicates that the value of $a$ which maximizes $F$ satisfies the relation

$$
a \sim\left(Q^{2} \sigma R^{-1}\right)^{1 / 3}\left[\log \left(R Q^{-1}\right)\right]^{-1 / 6} \text {. }
$$

The solutions can be obtained by matching asymptotic approximation in interior and two distinct regions near the boundary. In the interior of the layer the buoyancy and Lorentz forces balance, although the inertial or viscous forces could become significant too. The governing equations yield the following equations in this region

$$
\begin{aligned}
& D \omega=a^{2} H, \\
& \omega \theta=1,
\end{aligned}
$$

$$
-\alpha^{2} \omega+\theta-3 c \sigma^{-1}(F R)^{\frac{1}{2}} \omega D \omega+Q D H=0 \text {, }
$$


where it is assumed that

$(3.33 a)$

$(3.33 b)$

$(3.33 c)$
$Q \ll F R$,

$a^{4} \ll Q$,

$F R a^{6} \leq Q^{3} \sigma^{2}$.

(3.32a)-(3.32c) yield the following asymptotic results as $z \rightarrow 0$ :

$$
\omega=a z\left(2 Q^{-1} \log z^{-1}\right)^{\frac{1}{2}},
$$

$$
\theta=\omega^{-1} \text {, }
$$

$(3.34 c)$

$$
H=a^{-1}\left(2 Q^{-1} \log z^{-1}\right)^{\frac{1}{2}} \text {. }
$$

Closer to the boundary and adjacent to the interior is a thermal layer, in which termal conduction is significant and $\theta$ and $\omega$ satisfy the boundary conditions (2.6). We define $\eta=z / \delta$ as the variable in the layer, where $\delta$ is the thickness of the layer. The governing equations and matching conditions then yield the following equations

$$
\frac{d H}{d \eta}=0
$$

$$
\delta a^{2} H=\frac{d \omega}{d \eta},
$$

$$
a^{2} \theta=F R(1-\omega \theta) \omega,
$$

where it is assumed that

$$
F R A_{1}^{2}=a^{2},
$$

$$
(F R)^{\frac{1}{2}} A_{1} a^{2} \delta \ll \sigma Q \text {, }
$$

$$
1 \ll a \delta \ll A_{1}(Q)^{\frac{2}{2}},
$$

$$
A_{1}=a \delta\left(2 Q^{-1} \log \delta^{-1}\right)^{\frac{1}{2}} \text {. }
$$

$(3.35 a)-(3.35 c)$ are satisfied by 


$$
\omega=A_{1} \eta
$$

$$
H=A_{1} a^{-2} \delta^{-1}
$$

$$
\theta=A_{1}^{-1} n\left(1+\eta^{2}\right)^{-1}
$$

A further thinner layer near the boundary is needed to adjust the solution to satisfy the correct boundary condition on $H$. This is a new magnetic layer, in which Lorentz force is dominant in the momentum equation. We define $\xi=a z$ as the variable in the layer, where $a^{-1}$ turns out to be the thickness of the layer. We then find from (2.4) and matching conditio conditions that

$$
\left(\frac{d^{2}}{d \xi^{2}}-1\right) \frac{d H}{d \xi}=0,
$$

$$
a\left(\frac{d^{2}}{d \xi^{2}}-1\right) H+\frac{d \omega}{d \xi}=0 \text {, }
$$

$$
(F R)^{-1} a^{2}\left(\frac{d^{2}}{d \xi^{2}}-1\right) \theta+\omega=0 .
$$

(2.6) and (3.38a)-(3.38c) are satisfied by

$$
\omega=(a \delta)^{-1} A_{1} \xi
$$

$$
\theta=a \delta A_{1}^{-1} \xi
$$

$$
H=\left(\delta a^{2}\right)^{-1} A_{1}[1-\exp (-\xi)]
$$

The maximization of $F$ proceeds as before, and we find the following results:

$$
\delta=\pi Q\left[2 R \log \left(R Q^{-1}\right)\right]^{-1},
$$

$$
F=\left(\pi^{2} Q\right)^{-1}\left[2 R \log \left(R Q^{-1}\right)\right] \text {. }
$$

Various assumptions considered in this section lead to the following conditions for the validity of the solutions: 
(3.41a) $\left(R^{4} \sigma^{-1}\right)^{1 / 5}\left[\log \left(R Q^{-1}\right)\right]^{7 / 10} \ll Q \leq\left(R \sigma^{-1}\right)^{4 / 5}\left[\log \left(R Q^{-1}\right)\right]^{2 / 5}$,

$$
R^{-\frac{1}{4}}\left[\log \left(R Q^{-1}\right)\right]^{\frac{1}{2}} \ll \sigma \ll\left[\log \left(R Q^{-1}\right)\right]^{-\frac{1}{2}} \text {. }
$$

\section{Discussion}

The boundary layer analysis has shown that it is appropriate to divide the parameter space into four different regions. For a weak field, $F$ is independent of $Q$, but it depends strongly on $R$ and $\sigma$. It also increases with $\sigma$ and $R$. The stabilizing effect of the field is so small that the maximizing flow behaves as if there is no field. The horizontal scale of convection cells is also independent of $Q$. It is seen from the conditions (3.16) for the validity of the solutions that increasing the effects of the inertial forces can widen the range for a weak field. For example, as $\sigma$ approaches the lower limit $\left(R^{-1} \log R\right)^{1 / 9}$ in (3.16a), the upper limit for $Q$ approaches $R^{2 / 3}(\log R)^{2 / 15}$ which is the largest possible upper limit for $Q$, provided $\sigma$ lies in the range (3.16a). For a moderate field, the horizontal cells size decreases. $F$ increases with $R$ and $\sigma$ and decreases as $Q$ increases. It depends, however, weakly (through a logarithmic term) on $\sigma$. A new magnetic layer is formed, in which the Lorentz force is comparable in, magnitude with either inertial or buoyancy force. In contrast to the case of a weak field, it is seen from (3.25) that increasing the effects of the inertial force can not widen the range for a moderate field. This is due to that fact that for a moderate field the Lorentz force is significant enough to dominate over the inertial force. Thus, decreasing $\sigma$ in the ranges (3.25a), (3.25b) does not have significant effects on the flow properties and conditions. For a moderately strong field, the behavior of the flow is intermediate between cases of a moderate and strong field. The dependence of $F$ on $\sigma$ is weak and is essentially the same as in the case of a moderate field, but its dependence on $Q$ and $R$ is similar to the case of a strong field. The horizontal wave number now decreases with $Q$. For a strong field, the magnetic layer mentioned above disappears. However, a new thin magnetic layer is developed which is responsible to bring the fluctuating field to its correct boundary condition. $F$ decreases as $Q$ increases and is independent of $\sigma$.

In a recent study on magnetic convection, Riahi [6] investigated the 
case $c=0$. It is clear that in this case the dependence of the flow on $\sigma$ disappears and the results are believed to describe correctly the average properties of the flow at sufficiently large $\sigma$. Thus, it is not surprising if one finds some similarities between the results of the present study and the case $c=0$ only at sufficiently large $Q$. Indeed for sufficiently large $Q$, the dependence of $F$ on $Q$ and $R$ is qualitatively similar in both cases $c=0$ and $c \neq 0$. But the ranges for $\sigma$ and $Q$, the convection cells' size, dependence of the flow properties on $\sigma$ and other results are different.

The approximation $\tau \gg 1$ made in this paper which eliminates the advection of magnetic field and the onlinear interactions of fluctuating field and velocity in the induction equation is responsible for ruling out the possibilities of sub-critical instability (Busse, [1]) and magnetic flux expulsion by convection (Galloway, et al., [2]).

Numerical studies for three dimensional nonlinear magnetic convection have not yet been done, but the two dimensional problem has recently been studied numerically by Weiss [10] who found, for example, that for $R=10^{5}, \sigma=1, \tau=0.2$. for cells with length 0.5 that steady convection exists for $Q \leq 2.16 \times 10^{4}$ (in agreement with (3.41)) and for $Q \leq 1.25 \times 10^{2}$, the field no longer has any dynamical significance (in agreement with (3.16)). Our study is based on the maximized nonlinear asymptotic state $(R \gg 1)$ and $\tau \gg 1$, whereas Weiss' study is concerned with moderate values of $R$ and $\tau$. It is not expected that there will be many other similarities between the results of these two studies.

\section{References}

[1] F.H. Busse, "Nonlinear interaction of magnetic field and convection", J. Fluid Mech. 71 (1975), 193-206.

[2] D.J. Galloway, M.R.E. Proctor and N.O. Weiss, "Magnetic flux ropes and convection", J. Fluid Mech. 87 (1978), 243-261.

[3] P. Glansdorff and 1. Prigogine, "On a general evolution criterion in macroscopie physics", Physica 30 (1964), 351-374. 
[4] D.O. Gough, E.A. Speigel and Juri Toomre, "Modal equations for cellular convection", J. Fluid Mech. 68 (1975), 695-719.

[5] W.V.R. Malkus and G. Veronis, "Finite amplitude cellular convection", J. Fluid Mech. 4 (1958), 225-260.

[6] N. Riahi, "Some predictions from the mean field equations of magnetoconvection", Austral. J. Phys. 33 (1980), 47-58.

[7] P.H. Roberts, "On non-linear Benard convection", Non-equilibrizon thermodynamics, variational techniques and stability, 125-162 (Proc. Sympos. University of Chicago, 1965. University of Chicago Press, Chicago and London, 1966).

[8] A. Schlüter, D. Lortz and F. Busse, "On the stability of steady finite amplitude convection", J. Fluid Mech. 23 (1965), 129-144.

[9] Juri Toomre, D.O. Gough and E.A. Spiegel, "Numerical solutions of single-mode convection equations", J. Fluid Mech. 79 (1977), $1-31$.

[10] N.0. Weiss, "Magnetic fields and convection", Adv. Chem. Phys. 32 (1975), 101-107.

Department of Theoretical and Applied Mechanics,

College of Engineering,

University of 111 inois at Urbana-Champaign,

Urbana,

III inois 61801 ,

USA. 